\title{
A compreensão de situações - problema por alunos participantes de um projeto de Iniciação Científica Jr.
}

\section{The understanding of problem situations by students participating in a Jr. Scientific Initiation Project}

\section{La comprensión de situaciones problemáticas por parte de los estudiantes que participan en un proyecto de Iniciación Científica Jr.}

\author{
Andréia de Freitas Zompero (andreiazomp@uel.br) \\ Universidade Estadual de Londrina \\ Cínthia Hoch Batista de Souza (cinthiahoch@yahoo.com.br) \\ Universidade Estadual de Londrina \\ Bruna Lauana Crivelaro (crivelarolbruna@gmail.com) \\ Universidade Estadual de Londrina
}

Resumo: $O$ entendimento de procedimentos realizados em ciência pelos alunos da Educação Básica é um assunto que vem sendo debatido nos últimos anos. A identificação e o entendimento do problema a ser investigado é um dos conhecimentos fundamentais para que o estudante compreenda procedimentos da ciência e uma condição indispensável para o aluno compreender as características do conhecimento científico. Neste estudo tivemos por objetivo investigar a compreensão do problema, apresentado em determinadas situações, por alunos da Educação Básica que participaram de um projeto de Iniciação Científica Jr. durante um período de seis meses. Trata-se de uma pesquisa qualitativa e para obtenção dos dados os alunos responderam uma situação - problema antes de iniciarem o projeto de Iniciação Científica Jr. e outra após encerrarem o projeto. Os dados apontam que houve uma melhora discreta na compreensão dos elementos do problema após os estudantes terem participado do projeto, pois a aprendizagem de procedimentos acontece progressivamente. Consideramos que a permanência por mais tempo nas atividades do projeto poderá contribuir progressivamente para que os estudantes melhorem a capacidade de compreensão do problema a ser investigado em uma determinada situação.

Palavras-chaves: Iniciação científica Jr.; Procedimentos em Ciência; Identificação do problema.

Abstract: The understanding of procedures performed in science by students of Basic Education is a subject that has been debated in recent years. The identification and

Recebido em: 30/05/2021

Aceite em: 13/08/2021 
understanding of the problem to be investigated is one of the fundamental procedural knowledge for the student to understand procedures of science and an indispensable condition for the student to understand the characteristics of scientific knowledge. In this study we aimed to investigate the understanding of the problem presented in certain situations by students of Basic Education who participated in a project of Scientific Initiation Jr. for a six-month period. It is a qualitative research and to obtain the data the students answered a situation - problem before starting the project of Scientific Initiation Jr. and another after closing the project. The data indicate that there was a slight improvement in understanding the elements of the problem after having participated in the project, the learning of procedures happens progressively. We believe that longer stays in project activities can progressively help students to improve their understanding of the problem to be investigated in a given situation.

Keywords: Scientific initiation Jr.; Procedures in Science; Identification of the problem.

Resumen: La comprensión de los procedimientos realizados en ciencias por los estudiantes de Educación Básica es un tema que se ha debatido en los últimos años. La identificación y comprensión del problema a investigar es uno de los conocimientos fundamentales para que el alumno comprenda los procedimientos científicos y una condición indispensable para que el alumno comprenda las características del conocimiento científico. En este estudio se tuvo como objetivo investigar la comprensión del problema, presentado en determinadas situaciones, por estudiantes de Educación Básica que participaron en un proyecto de Iniciación Científica Jr. durante un período de seis meses. Es una investigación cualitativa y para obtener los datos, los estudiantes respondieron una situación: un problema antes de comenzar el proyecto de Iniciación Científica Jr. y otro después de terminar el proyecto. Los datos indican que hubo una leve mejoría en la comprensión de los elementos del problema luego de que los estudiantes participaron en el proyecto, ya que el aprendizaje de los procedimientos ocurre progresivamente. Creemos que la permanencia en las actividades del proyecto por más tiempo puede contribuir progresivamente a que los estudiantes mejoren su capacidad para comprender el problema a investigar en una situación determinada.

Palabras-clave: Iniciación científica Jr.; Procedimientos en ciencia; Identificación del problema.

\section{INTRODUÇÃO}

A sociedade em que vivemos exige a formação de pessoas que estejam preparadas para lidar com problemas de natureza diversas. A escola, por meio das disciplinas do currículo, tem papel fundamental em oportunizar o desenvolvimento de competências aos estudantes de modo a prepará-los para a sociedade. Sabemos que cada área do conhecimento tem suas especificidades para contribuir na formação do aluno, portanto, 
o ensino das diferentes disciplinas deve também ser ministrado de maneira a atender essas particularidades disciplinares.

As Ciências da Natureza apresentam componentes que lhes são peculiares. O ensino das disciplinas voltadas a essa área não pode ser restrito apenas ao trabalho de conteúdos de natureza declarativa. O entendimento das Ciências da Natureza necessita também oportunizar aos alunos a compreensão e domínio de processos e práticas da investigação científica. Essa é uma das competências propostas na Base Nacional Comum Curricular (BRASIL, 2017).

Assim, concordamos com Meléndez (2012) que a ciência não pode ser ensinada sem a dimensão procedimental, pois a aprendizagem desse tipo de conhecimento contribui para que o aluno seja capaz de resolver problemas e situações distintas da vida cotidiana. Por isso, o conhecimento procedimental apresenta-se cada vez mais relevante no ensino das disciplinas da área de Ciências da Natureza.

Zabala (1998, p.44) afirma que os procedimentos "são suficientemente diferentes para que a aprendizagem de cada um deles tenha características bem específicas". O autor classifica os procedimentos em três tipos: motor/cognitivo; poucas ações/múltiplas ações; algorítmico/heurístico.

A aprendizagem de procedimentos científicos envolve a realização de tarefas relacionadas com várias dimensões como, por exemplo, desenvolver processos práticos, realizar atividades experimentais e saber transferir o conhecimento para outros contextos (PRO, 2013). Nesse sentido é relevante entender que a pessoa alfabetizada cientificamente além da compreensão de conteúdos de natureza declarativa é também capaz de fazer e desenvolver ações. Conforme Sternberg (2000), o conhecimento declarativo é um corpo organizado de informações factuais e pode ser representado por meio de palavras ou por símbolos, através de conceitos ou esquemas. A compreensão conceitual do conhecimento declarativo passa necessariamente pela psicologia cognitiva, na medida em que o mesmo se estabelece com base nesta matriz teórica (HEGENBERG, 2002).

O conteúdo procedimental é conforme afirmam Coll e Valls (2000, p. 77), “o conhecimento de um conjunto de ações ordenadas, orientadas para a consecução de uma meta". Diversos autores e documentos de ensino têm ressaltado a necessidade de que os 


\section{Edição Especial: Pesquisa na Pós-Graduação em Ensino de Ciências}

\section{Vol. 4, n. 5.2021}

ISSN: $2595-4520$

estudantes aprendam conteúdos de natureza procedimental, como os Parâmetros

Curriculares Nacionais (1998), Pozo e Crespo (2009), Zabala e Arnau (2010), National

Research Council (2012) e também marcos teóricos da avaliação internacional de estudantes (Programa Internacional de Avaliação de Estudantes - OECD - PISA, 2013; 2015). Esses documentos ressaltam a importância para os estudantes dos conhecimentos e procedimentos padrões utilizados na investigação científica, os quais compõem os conteúdos procedimentais.

Clement e Terrazzan (2011) apontam que uma justificativa relevante a favor da inclusão de conteúdos procedimentais nos currículos escolares é a de que as pessoas, ao concluírem a escolarização básica, saibam fazer o maior número de atividades possíveis com o conhecimento construído ao longo de seu processo de escolarização. Nesse sentido, considerando as especificidades da área de Ciências da Natureza, os alunos devem aprender conhecimentos do tipo procedimental que são próprios dessa área de conhecimento.

Sabemos que a construção do conhecimento científico é feita com base no questionamento, na dúvida, no problema. Conforme afirma Bachelard (1996) o conhecimento científico se desenvolve a partir de questionamentos. Por isso, entender a importância do problema é uma condição indispensável para o aluno compreender as características do conhecimento científico. Os conteúdos procedimentais em ciência passam necessariamente pelo reconhecimento do problema. Assim, defendemos que a identificação e o entendimento do problema a ser investigado é um dos conhecimentos procedimentais fundamentais para que o estudante compreenda procedimentos da ciência.

Consideramos que a escola pode oportunizar diferentes práticas, além daquelas utilizadas em sala de aula, para possibilitar aos estudantes a aprendizagem de conteúdos procedimentais direcionados à resolução de problemas. Uma dessas práticas que temos defendido em nossos estudos é a proposta da Iniciação Científica Jr. no Ensino Médio, pelo motivo de os alunos terem oportunidade de vivenciarem procedimentos que são próprios da ciência. Projetos de Iniciação Científica Jr. são atualmente oportunizados por muitas escolas, tanto públicas como particulares e há, até mesmo, incentivo por meio de bolsas do CNPq a alunos participantes, porém, conforme Tadiello e Robaine 
(2020) as instâncias governamentais ainda destinam poucos recursos para a educação brasileira.

Assim, o que buscamos responder neste estudo é qual a compreensão do problema apresentada por alunos do Ensino Médio que participaram de um projeto de Iniciação Científica Jr. Para tanto, fizemos uma tomada de dados com os estudantes no início do projeto e após finalizarem para compararmos o desempenho apresentado por eles. Concordamos com Solino e Sasseron (2018), quanto à necessidade de discutir a significação do problema para os alunos, visto que ele é a mola propulsora em atividades didáticas de cunho investigativo (CARVALHO et al., 1998). Sabemos que a temática de resolução de problemas se constituiu em um campo tradicional de pesquisa na área de educação em ciências (CLEMENT; TERRAZZAN, 2011). Assim, diversos estudos em Ciências da Natureza têm sido realizados sobre a resolução de problemas pelos estudantes. No entanto, consideramos que entender o problema, isto é, o que se busca em uma investigação, é prioritário nessa prática para que o aluno possa, primeiramente, atribuir significado ao problema antes de iniciar a investigação. Por isso, temos por objetivo neste estudo investigar a compreensão acerca do problema em alunos do Ensino Médio que participaram de um projeto de Iniciação Científica Jr. no intuito de averiguar se o projeto contribuiu para que os estudantes pudessem identificar e compreender uma determinada situação - problema com mais clareza.

\section{MARCOS TEÓRICOS}

Os conteúdos procedimentais apresentam natureza diferente dos conteúdos declarativos. Conforme Pozo e Crespo (2009, p. 47), “os procedimentos não se aprendem nem se ensinam como os outros conteúdos e, portanto, o que professores e alunos precisam fazer para conseguir superar as dificuldades no seu aprendizado é diferente do tradicional explicar e escutar". Neste sentido Coll e Valls (2000) apontam que o conhecimento procedimental apresenta como características: i) uma atuação; ii) não uma atuação qualquer, mas ordenada; e iii) orientada para a consecução de uma meta. Os autores expõem determinados verbos que consideram de natureza procedimental como, por exemplo, manejar, usar, construir, aplicar, coletar, observar, 
experimentar, elaborar, simular, demonstrar, planejar, avaliar, representar, analisar, identificar. Portanto, esse tipo de conteúdo refere-se ao fazer, às ações.

Para aprender conteúdos procedimentais é importante o fazer, exercitar determinada ação. Porém, não basta que sejam realizadas uma vez, é necessário várias vezes para que o aluno possa dominá-la, tantas vezes quantas forem necessários (ZABALA, 2010). Nesse sentido, é importante ressaltar que a aprendizagem de procedimentos não envolve apenas explicar e escutar. Por isso, a educação científica se obriga a adotar como um de seus objetivos principais a prática de ajudar os alunos a aprender e a fazer ciências (POZO; CRESPO, 2009).

Um dos procedimentos que tem sido muito incentivado na educação científica é a resolução de problemas. Quanto a isso, Pozo e Crespo (2009) propõem uma classificação para essa finalidade. Dentre essa classificação, as categorias aquisição e interpretação da informação referem-se ao entendimento do problema. Para cada uma dessas categorias os autores apresentam os procedimentos que estão envolvidos, conforme o Quadro 1 apresentado abaixo:

Quadro 1 - Conhecimentos procedimentais.

\begin{tabular}{|c|c|}
\hline Categoria & Procedimento \\
\hline Aquisição da informação & $\begin{array}{c}\text { Observação, seleção e busca da } \\
\text { informação }\end{array}$ \\
\hline Interpretação da informação & $\begin{array}{c}\text { Decodificação da informação, busca } \\
\text { de modelos para interpretar a } \\
\text { informação }\end{array}$ \\
\hline
\end{tabular}

Fonte: Adaptado de Pozo e Crespo (2009).

Uma outra classificação que admitimos ser mais completa é a proposta por Pro (1998). O autor classifica os procedimentos em investigativos e manipulativos. Nesse caso, referindo-se às habilidades investigativas e destrezas manuais. Interessa-nos as habilidades de investigação. Dentre essas habilidades, a primeira indicada pelo autor refere-se à identificação do problema. Estão implícitas nessa habilidade, segundo o 
autor, o conhecimento dos motivos do problema, identificação das partes do problema, das variáveis e abordagem de questões.

A ação de identificar remete a distinguir e reconhecer particularidades. Portanto, para a compreensão do enunciado do problema é necessário a identificação dos seus elementos constituintes, pois sem entendê-lo não há uma resolução coerente. Nesse sentido, Costa e Moreira (2001) afirmam que a estrutura cognitiva do aluno desempenha papel preponderante na resolução de problemas, considerando que a busca de solução de qualquer problema envolve uma readaptação da experiência prévia da pessoa frente às demandas da nova situação problemática a ser enfrentada. Os autores afirmam que apenas entender um enunciado não significa que o estudante saberá resolver o problema, mas não entendê-lo é a primeira condição para não avançarmos no conhecimento que ele poderia gerar.

De acordo com Pro (2013), a aprendizagem dos conteúdos procedimentais é necessária para o aluno crescer intelectualmente e desenvolver capacidades, potencialidades individuais e atender suas necessidades como cidadão. Nesse sentido, a aprendizagem desse tipo de conteúdo oportuniza ao aluno o desenvolvimento de habilidades cognitivas.

Encontramos na literatura uma outra classificação proposta por Zoller (2001; 2012; 2013) relativa às habilidades cognitivas que o autor classifica em Algorítmicas (ALG), de baixa ordem (Lower order cognitive skills - LOCS) e de alta ordem (Higher order cognitive skills - HOCS) e que adaptamos para analisar a compreensão dos alunos sobre o problema em uma determinada investigação. De acordo com o referido autor, o aluno desenvolve habilidades cognitivas de baixa ordem (LOCS) e as de alta ordem (HOCS). As habilidades cognitivas de baixa ordem são, por exemplo, lembrar/recordar uma informação e aplicar conhecimentos em situações triviais ou para resolver exercícios. Ao contrário, as habilidades cognitivas de alta ordem, o autor classifica como aquelas relacionadas com a resolução de problemas não familiares, capacidade de fazer conexões e pensamento avaliativo, o que pode ser promovido por práticas que exijam uma atividade intelectual mais elaborada do estudante.

\section{METODOLOGIA}

Recebido em: 30/05/2021

Aceite em: 13/08/2021 
Trata-se de uma pesquisa qualitativa do tipo descritiva e interpretativa (GIL, 2008). Após aprovação do projeto pelo Comitê de Ética em Pesquisa com Seres Humanos- CAAE 60351316.3.0000.0108, o estudo foi realizado com a participação de dez alunos do segundo ano do Ensino Médio de uma escola pública estadual da cidade de Londrina, PR, que participaram pela primeira vez de um projeto de Iniciação Científica Jr. Uma vez por semana, no contraturno escolar, durante o período de seis meses, os alunos participaram das atividades de um projeto de pesquisa desenvolvido em um Programa de Pós-Graduação em Ciência e Tecnologia de Leite e Derivados, em uma Instituição de Ensino Superior Particular. Os alunos foram orientados por uma docente e auxiliados pela técnica do laboratório e alunas do programa (duas mestrandas e duas alunas de iniciação científica, que também participaram do projeto). O envolvimento dos alunos na Iniciação Científica Jr. compreendeu duas etapas: I atividades práticas e II - momentos de interação e discussão. Durante a realização das atividades práticas os alunos produziram, em escala piloto, um lote de iogurte natural, seguindo um roteiro específico para essa atividade. Assim, eles foram os responsáveis pelo preparo dos materiais e pela produção do alimento, bem como pela realização de análises físico-químicas durante e após a produção. Nesta parte prática os alunos tiveram contato com equipamentos e técnicas que exigiram compreensão dos métodos, atenção e precisão, para que conseguissem obter o produto proposto, bem como resultados analíticos confiáveis, ou seja, que fossem compatíveis com o preconizado na legislação brasileira para caracterização de iogurtes.

Concomitantemente às atividades práticas, os alunos, juntamente com as mestrandas, discutiam os dados obtidos nos experimentos, momento em que tinham oportunidade de argumentar e colocar questionamentos. As discussões eram realizadas após o término das práticas laboratoriais, uma vez que os alunos estavam com os resultados finais em mãos.

As discussões sobre os resultados obtidos levaram os estudantes a pensarem, por exemplo, sobre as alterações ocorridas durante o processo fermentativo que transformou o leite em iogurte. No Quadro 2 estão apresentadas as atividades práticas, os resultados obtidos pelos alunos e a compreensão deles sobre a investigação. 
Quadro 2 - Atividades práticas e de investigação científica realizadas pelos alunos durante a participação no projeto de pesquisa.

\begin{tabular}{|c|c|c|}
\hline Atividade prática & $\begin{array}{c}\text { Resultado } \\
\text { observado pelos } \\
\text { alunos }\end{array}$ & Aspectos discutidos \\
\hline $\begin{array}{l}\text { Análise da acidez do } \\
\text { leite durante a } \\
\text { produção do iogurte }\end{array}$ & $\begin{array}{l}\text { Aumento } \\
\text { progressivo da } \\
\text { acidez a cada } \\
\text { medida. }\end{array}$ & $\begin{array}{l}\text { Os micro-organismos } \\
\text { adicionados para } \\
\text { produção do iogurte } \\
\text { estavam realizando } \\
\text { fermentação do açúcar } \\
\text { do leite (lactose) e } \\
\text { produzindo ácidos, } \\
\text { qu foram os } \\
\text { responsáveis pela } \\
\text { alteração dos valores } \\
\text { de acidez. }\end{array}$ \\
\hline $\begin{array}{l}\text { Verificação da textura } \\
\text { do leite durante a } \\
\text { produção do iogurte. } \\
\text { a InSignare }\end{array}$ & $\begin{array}{l}\text { Produto apresentava } \\
\text { mudança na textura. }\end{array}$ & $\begin{array}{l}\text { O ácido produzido } \\
\text { pelos micro- } \\
\text { organismos alterou a } \\
\text { estrutura proteica do } \\
\text { leite, formando uma } \\
\text { rede responsável pela } \\
\text { característica sólida } \\
\text { do iogurte. }\end{array}$ \\
\hline $\begin{array}{l}\text { Análise do aroma do } \\
\text { leite durante a } \\
\text { produção do iogurte. }\end{array}$ & $\begin{array}{l}\text { Produto apresentava } \\
\text { aroma diferenciado a } \\
\text { cada verificação. }\end{array}$ & $\begin{array}{l}\text { Característica } \\
\text { esperada, uma vez que } \\
\text { além de ácidos os } \\
\text { micro-organismos } \\
\text { também produziram } \\
\text { compostos voláteis } \\
\text { aromáticos que foram } \\
\text { percebidos pelos }\end{array}$ \\
\hline
\end{tabular}




\begin{tabular}{|l|l|l|}
\hline Verificação da textura & O iogurte se tornou & Olunos. \\
do iogurte batido com & líquido. & $\begin{array}{l}\text { Oncorporação da polpa } \\
\text { polpa de fruta. }\end{array}$ \\
de fruta rompeu a rede \\
de proteína formada \\
anteriormente, \\
fazendo com que o \\
produto apresentasse \\
textura diferente.
\end{tabular}

Fonte: Dados da pesquisa.

Para obtenção dos dados e investigarmos a compreensão dos alunos sobre o problema, os estudantes responderam a duas situações - problema. A primeira foi aplicada antes de os estudantes iniciarem as atividades do projeto e a segunda aplicada duas semanas após terem finalizado as atividades. As duas atividades avaliavam as mesmas habilidades.

A atividade 1 foi sobre osmose: Suponha que você precise desidratar folhas de alface. Para realizar esse processo você tem alguns materiais a sua disposição como bacias com água, sal, vinagre, óleo, pimenta e folhas de alface. Porém, você precisa saber com certeza qual dos ingredientes acima é responsável por esse processo. Responda: Qual o problema está sendo investigado?

A atividade 2 foi referente ao cultivo de samambaias: As samambaias são plantas que para se desenvolverem necessitam de certas condições de luminosidade e umidade. Imagine agora que você vai trabalhar com o cultivo de samambaia e, por isso, precisa saber em que condições de umidade e de luminosidade as samambaias se desenvolvem melhor. Responda: Qual é o problema que você está buscando resolver nessa atividade?

\section{RESULTADOS E DISCUSSÃO}

Recebido em: 30/05/2021

Aceite em: 13/08/2021 
As situações-problema eram compostas por quatro questões, porém neste estudo, analisamos as respostas dadas somente à primeira pergunta sobre qual seria o problema a ser investigado em ambas as situações. Para análise das respostas dos alunos, tomamos por base a fundamentação de Pro (1998) e a classificação de Suart e Marcondes (2009), que propõem determinados níveis para indicar as habilidades LOCS e HOCS dos estudantes. Assim, estabelecemos quatro níveis para indicar a compreensão dos estudantes sobre o problema investigado nas situações 1 e 2 que lhes foram apresentados.

No nível 1 estão incluídos os alunos que não reconhecem a situação - problema e não identificam com clareza qual o questionamento proposto apresentado na situação problema, identificada segundo Zoller (2012) como algorítmica. Na categoria LOCS estão organizados os níveis 2 e 3 . Ambos indicam que o estudante reconhece a situação - problema, porém, no caso do nível 2 foram incluídos os estudantes que não identificam com clareza o que deve ser buscado e no nível 3 aqueles que reconhecem a situação - problema e também têm clareza do que deve ser buscado.

A categoria indicada como HOCS está no nível 4 e nela estão relacionados alunos que selecionam informações relevantes para identificar o problema. Dentre essas informações classificamos as respostas dos alunos que identificaram as variáveis apresentadas no problema. As categorias utilizadas neste estudo foram adaptadas com base no estudo de Suart e Marcondes (2009). O Quadro 3 apresenta a classificação das respostas dos estudantes participantes da Iniciação Científica Jr. Utilizamos as iniciais dos nomes de cada estudante.

Quadro 3 - Categorização das respostas dos estudantes.

\begin{tabular}{|c|c|c|c|}
\hline Níveis & Categoria & $\begin{array}{c}\text { Atividade 1 } \\
\text { (alunos) }\end{array}$ & $\begin{array}{c}\text { Atividade } 2 \\
\text { (alunos) }\end{array}$ \\
\hline \multicolumn{4}{|c|}{ Categoria Algorítmica } \\
\hline N1 & $\begin{array}{l}\text { Não reconhece o problema a } \\
\text { ser investigado. }\end{array}$ & $\begin{array}{l}\text { Am, Ga, } \\
\text { Ma }\end{array}$ & $\begin{array}{l}\text { Não } \\
\text { apresenta }\end{array}$ \\
\hline \multicolumn{4}{|c|}{ Categoria LOCS } \\
\hline
\end{tabular}

Recebido em: 30/05/2021

Aceite em: 13/08/2021 


\begin{tabular}{|c|c|c|c|}
\hline $\mathrm{N} 2$ & $\begin{array}{l}\text { Reconhece a situação - } \\
\text { problema e não identifica } \\
\text { com clareza o que deve ser } \\
\text { buscado. }\end{array}$ & $\mathrm{Lu}, \mathrm{Br}, \mathrm{Va}$ & $\begin{array}{l}\text { Am, Lu, } \\
\text { Ma, La, Ga }\end{array}$ \\
\hline N3 & $\begin{array}{l}\text { Reconhece a situação - } \\
\text { problema e identifica com } \\
\text { clareza o que deve ser } \\
\text { buscado. }\end{array}$ & $\begin{array}{l}\text { Gi, Me, Ya, } \\
\text { La }\end{array}$ & $\begin{array}{l}\text { Gi, Va, Me, } \\
\text { Br, Ya }\end{array}$ \\
\hline \multicolumn{4}{|c|}{ Categorias HOCS } \\
\hline N4 & $\begin{array}{l}\text { Seleciona informações } \\
\text { relevantes para identificar o } \\
\text { problema. }\end{array}$ & $\mathrm{La}, \mathrm{Me}, \mathrm{Gi}$ & $\begin{array}{l}\mathrm{Ya}, \mathrm{Br}, \mathrm{Me} \text {, } \\
\mathrm{Gi}\end{array}$ \\
\hline
\end{tabular}

Fonte: Dados da pesquisa.

No nível 1 estão classificados os alunos que não reconhecem a situação problema. Na atividade 1, que corresponde à situação - problema 1, identificamos os alunos Am, Ga e Ma que não a reconheceram. Algumas respostas para essa pergunta foram:

"Se por acaso tirar a alface da colheita por exemplo e deixar em algum outro lugar já ira desidratar sozinho” (Am).

"Trata de culinária, na culinária procura-se ter conhecimentos químicos, o problema acima pode ser uma solução cotidiana” (Ga).

"O problema que está sendo investigado desta maneira, o vinagre ele tem características relacionadas a acidez, o óleo tem características de não se misturar com líquidos de carga diferente e o sal tem características de conservar alimentos" (Ma).

Na atividade 2, que corresponde à situação - problema 2 e que ocorreu após finalizar o projeto, todos os alunos identificaram a situação - problema, no entanto, alguns não tiveram clareza na compreensão. Esse fato pode ser observado nos níveis $2 \mathrm{e}$ 3. Nesses dois níveis estão classificadas as atividades do tipo LOCS. Os alunos AM, 
GA e MA que não haviam reconhecido o problema na atividade 1, percebemos que na atividade 2 conseguem reconhecer o problema, mas ainda não identificam com clareza o que deve ser buscado, conforme respostas abaixo.

"Encontrar um local apropriado para o cultivo" (AM).

"Descobrir as condições de ambiente que são favoráveis e adequadas para o bom desenvolvimento e reprodução da planta” (GA).

"O problema é localizar esse local e as pessoas com experiência adequada para se realiza essa atividade” (MA).

Os alunos $\mathrm{Br}$ e $\mathrm{Va}$, na atividade 1, identificaram o problema, mas não tiveram clareza quanto ao que deveria ser investigado. Na atividade 2 foi possível notar que ambos apresentaram entendimento do que deveriam buscar no problema, conforme respostas abaixo.

Atividade 1: "A reação de desidratação das folhas de alface” (Br); "A reação de desidratação das folhas de alface" (Va).

Atividade 2: "Estou buscando um lugar com umidade, luminosidade melhor para ela se desenvolver" (Br); "O primeiro problema é a baixa umidade e a outra a luminosidade" (Va).

Assim, notamos nesses dois níveis que houve avanços no entendimento dos alunos quanto ao reconhecimento do problema.

Quanto ao nível 4, sobre a seleção de informações relevantes para resolver o problema, foram classificadas as respostas dos alunos que identificaram variáveis, por considerarmos essa compreensão essencial para identificar o problema com clareza e possibilitar o posterior teste de hipóteses e elaboração de um plano de trabalho para resolvê-lo. Comparando-se as atividades 1 e 2 observamos que os estudantes $\mathrm{Ya}$ e $\mathrm{Br}$ tiveram um melhor desempenho na atividade 2 com relação à percepção de variáveis, conforme respostas a seguir.

Atividade 1: "Uma forma de desidratar folhas de alface” (Ya).

Atividade 2: "Uma melhor condição (ou forma), para que a samambaia se desenvolva melhor, ou seja, achar medida certa de umidade e luminosidade" (Ya).

Recebido em: 30/05/2021

Aceite em: 13/08/2021 


\section{A aluna indica com clareza na atividade 2 as variáveis umidade e} luminosidade. Tal fato mostra que a estudante conseguiu selecionar informações relevantes contidas no enunciado do problema. O mesmo ocorreu com o aluno $\mathrm{Br}$ ao responder estou buscando um lugar com umidade, luminosidade melhor para ela se desenvolver em que o estudante expõe as variáveis umidade e luminosidade na situação 2, e não as identifica na situação - problema na atividade 1 , mencionando apenas que "A reação de desidratação das folhas de alface".

No entanto, a estudante La apresentou melhor desempenho na atividade 1, o que pode ser observado nas respostas da aluna em ambas atividades a seguir:

Atividade 1: "Desidratar folhas de alface, com os elementos indicados para uso do processo" (La).

Atividade 2: "Cuidar das samambaias porque irei cultivá-las” (La).

Comparando as respostas em ambas as atividades, os dados apontam que os estudantes tiveram um desempenho discretamente superior na atividade 2 em relação à atividade 1 quanto à identificação do problema nas situações que lhes foram apresentadas. Consideramos relevante o fato de todos os estudantes terem identificado o problema na atividade 2 e o fato de os estudantes Am, Ga e Ma terem suas respostas da atividade 2 classificadas no nível 2, mesmo sem terem clareza do problema a ser investigado. Isso demonstra a manifestação de habilidades do tipo LOCS e um avanço em relação aos resultados da atividade 1. Outro avanço possível de observar foi quanto ao desempenho dos alunos Br, Va e Ya em relação à atividade 2, pois suas repostas a esta atividade puderam ser classificadas nos níveis 3 e 4. Além disso, os alunos Br e Ya conseguiram selecionar informações na atividade 2 quando identificaram as variáveis do problema, demonstrando habilidades do tipo HOCS.

Não notamos avanços na qualidade das respostas dos alunos $\mathrm{Me}$ e Gi, pois permaneceram no mesmo nível em ambas as atividades. A aluna La apresentou melhor desempenho na resolução da atividade 1 . Inferimos que nesse caso, a participação no projeto de Iniciação Científica Jr., para essa aluna, não contribuiu para a melhor compreensão do problema em uma atividade a ser investigada. Além dos resultados observados para os alunos participantes da pesquisa, é importante destacar a experiência oferecida às alunas do programa de pós-graduação que participaram do projeto. $\mathrm{O}$ Recebido em: 30/05/2021

Aceite em: 13/08/2021 
convívio com os alunos do Ensino Médio proporcionou a essas alunas a oportunidade de realizarem atividades de ensino, uma vez que as atividades práticas bem como os encontros para discussão dos dados obtidos foram situações organizadas e acompanhadas pelas pós-graduandas. Essa experiência certamente foi de grande relevância, visto que o aluno de pós-graduação deve se preparar para atividades de ensino, além da pesquisa. Atividades realizadas junto de alunos do Ensino Médio, como as descritas neste estudo, enriquecem a formação dos pós-graduandos, que podem ensinar e discutir os dados em uma linguagem específica para esses alunos, porém, mantendo a informação e o rigor científico.

\section{CONCLUSÃO}

Neste trabalho analisamos o desempenho de um grupo de alunos do Ensino Médio quanto à compreensão do problema a ser investigado, por meio de respostas para duas situações-problema que tiveram contato, identificadas neste estudo respectivamente como atividade 1 e atividade 2. Admitimos que a compreensão do problema é o primeiro passo para o desenvolvimento de uma investigação. Podemos inferir que a participação no projeto de Iniciação Científica Jr. possibilitou o desenvolvimento da capacidade de identificar problemas aos alunos aqui mencionados. Consideramos que o entendimento do problema a ser investigado em uma determinada situação é parte do que é estabelecido como conhecimento procedimental. No entanto sabemos, como afirmam Pozo e Crespo (2009) que a aprendizagem de procedimentos acontece progressivamente, isto é, o aluno não aprende um procedimento por completo num primeiro momento, mas, amplia sua aprendizagem ao longo do tempo. Além disso, a aprendizagem de procedimentos poderá favorecer a atribuição de significados para outros conhecimentos (PRO, 2013).

Os alunos participaram do projeto durante um período de seis meses. Assim, admitimos que a permanência por mais tempo nas atividades do projeto poderá contribuir progressivamente para que os estudantes melhorem a capacidade de compreensão do problema a ser investigado em uma determinada situação.

Sabemos que os conteúdos de natureza conceitual em Ciências são priorizados pelos professores em relação aos procedimentais e que os docentes também apresentam Recebido em: 30/05/2021 Aceite em: 13/08/2021 
dificuldades em ministrá-los, conforme aponta Meléndez (2012). Além disso, os conteúdos de natureza procedimental não são comuns nas práticas dos professores porque muitos não tiveram oportunidade de aprendê-los durante sua formação, conforme apontam Munhõs e Charro (2017). Dessa maneira, os projetos de Iniciação Científica Jr. constituem-se em uma rica oportunidade para aprendizagem dos alunos tanto no que se referem aos conteúdos conceituais como aos procedimentais.

\section{REFERÊNCIAS}

BACHELARD, G. A formação do espírito científico. Rio de Janeiro: Contraponto, 1996.

BRASIL. Ministério da Educação. Base Nacional Comum Curricular: educação é a base. 2017. Disponível em:

http://cnebncc.mec.gov.br/docs/BNCC_Educacao_Infantil_e_Ensino_Fundamental.pdf >. Acesso em: 5 jul. 2018.

BRASIL. Ministério da Educação. Parâmetros Curriculares Nacionais. Ciências Naturais. Brasília: MEC, 1998.

CARVALHO, Anna Maria Pessoa de. et al. Ciências no Ensino Fundamental: o conhecimento físico. São Paulo: Scipione, 1998. (Pensamento e Ação no Magistério).

CLEMENT, L.; TERRAZZAN, E. A. Atividades didáticas de resolução de problemas e o ensino de conteúdos procedimentais. Revista Electrónica de Investigación en

Educación en Ciências, v. 6, n. 1, p. 87-101, 2011. Disponível em:

〈http://ppct.caicyt.gov.ar/index.php/reiec/article/view/7463>. Acesso em: 20 abr. 2021.

COLL, C.; VALLS, E. A aprendizagem e o ensino de procedimentos. In: COLL, C. Os conteúdos na reforma: ensino e aprendizagem de conceitos, procedimentos e atitudes. Porto Alegre: Artes Médicas, 2000.

COSTA, S. S. C.; MOREIRA, M. A. A resolução de problema como um tipo especial de aprendizagem significativa. Caderno Brasileiro de Ensino de Física, v. 18, n. 3; p. 263-277, 2001. Disponível em:

<https://periodicos.ufsc.br/index.php/fisica/article/view/6663/19039>. Acesso em: 20 abr. 2021.

GIL, Antonio Carlos. Como elaborar projetos de pesquisa. 4. ed. São Paulo: Atlas, 2008.

HEGENBERG, L. Saber de e saber que: alicerces da racionalidade. Petrópolis: Vozes, 2002.

MELÉNDEZ, A. Z. Los contenidos procedimentales en el proceso de enseñanzaaprendizaje (el caso de una escuela en Mendoza República de Argentina). Tese. Departamento de Didáctica de las Ciencias Experimentales, Universidad de Granada. Granada, p.417, 2012. 417 p. Disponível em <http://hdl.handle.net/10481/23766>.

Acesso em: 20 abr. 2021.

Recebido em: 30/05/2021

Aceite em: 13/08/2021 
MUÑOZ, J. I.; CHARRO, E. Los ítems PISA como herramienta para el docente em la identificaccion de los conocimientos y habilidades cientificas. Revista Eureka sobre Enseñanza y Divulgación de las Ciencias, v. 14, n. 2, p. 317-338, 2017.

NATIONAL RESEARCH COUNCIL. A framework for K-12 science education: practices, crosscutting concepts, and core ideas. Washington, DC: The National Academies Press, 2012.

OECD-PISA. (2015). Draft Science Framework. Paris, 2013. Paris. Disponível em <http://www.oecd.org/pisa/pisaproducts/Draft\%20PISA\%202015\%20Science\%20Fram ework\%20.pdf >. Acesso em: 19 mar. 2018.

PRO; B. A. Enseñar procedimientos: por qué e para qué. Alambique: Didáctica de las Ciencias Experimentales, n, 73, p. 69-76, 2013.

PRO; B. A. ¿Se poden enseñar contenidos procedimentales em clases de ciências?

Enseñansa de las Ciencas, v. 16, n. 1, p. 21-41, 1998.

POZO, J. I.; CRESPO, M. A. G. A aprendizagem e o ensino de Ciências: do conhecimento cotidiano ao conhecimento científico. Porto Alegre: Artmed, 2009.

SOLINO, A. P.; SASSERON, L. H. Investigando a significação de problemas em sequência didática investigativa. Investigações em Ensino de Ciências, v. 23, n. 2, p. 104-129, 2018.

STERNBERG, R. J. Psicologia cognitiva. Porto Alegre: Artmed, 2000.

SUART, R. C.; MARCONDES, M. E. R. A manifestação de habilidades cognitivas em atividades experimentais investigativas no ensino médio de Química. Ciências \& Cognição, v. 14, n. 1, p. 50-74, 2009.

TADIELLO, Rafaela Bressan; ROBAINA, José Vicente Lima; Análise das percepções docentes e discentes sobre a Sequência de Ensino Investigativa. RIS -Revista Insignare Scientia. Vol. 3, n. 1. Jan./Abr. 2020

ZABALA, A. A Prática Educativa: como ensinar. Porto Alegre: Artmed, 1998.

ZABALA, A.; ARNAU, L. Como aprender e ensinar competências. Porto Alegre: Artmed, 2010.

ZOLLER, U. Alternative assessment as (critical) means of facilitating hocs-promoting teaching and learning in chemistry education. Chemistry Education Research and Practice, v. 2, n. 1, p. 9-17, 2001.

ZOLLER, U. Science education for global sustainability: what is necessary for teaching, learning and assessment strategies? Journal of Chemical Education, v. 89, p. 297-300, 2012.

ZOLLER, U. Science, technology, environment, society (STES) literacy for sustainability: what should it take in chem/science education? Educación Química, v. 24, n. 2, p. 207-214, 2013.

Recebido em: 30/05/2021

Aceite em: 13/08/2021 